\title{
CONSIDERACIONES PRELIMINARES PARA UN ESTUDIO DEL GAITANISMO EN LA CIUDAD DE CALI
}

\author{
Esteban Morera Aparicio**
}

\begin{abstract}
Resumen
El presente artículo pretende mostrar una serie de obras que se pueden considerar como indispensables para el estudio del fenómeno gaitanista en la ciudad de Cali. Además busca revelar cómo a pesar de la importancia que tuvo este movimiento en la ciudad y la ciudad en este movimiento, la producción y discusión académica en este sentido han sido muy limitadas, situación que cuenta con un panorama alentador a la luz de los últimos trabajos que se han venido desarrollando.
\end{abstract}

Palabras Clave: gaitanismo, Violencia, violencia bipartidista, Santiago de Cali.

\begin{abstract}
This article offers a series of documents that can be considered as indispensable for the study of the "gaitanista" phenomenon in Cali. It also seeks to show how, despite its important role of this movement in the city and of the city in this movement, the production and academic discussion in this regard have been very limited, a situation that shows a change, a promising horizon because of recent works that have been developed.
\end{abstract}

Key words: gaitanismo, Violencia, bipartisan violence, Santiago de Cali.

A inicios del siglo $X X$, el país vivió una serie de transformaciones gracias a su consolidación en el mercado internacional. El comercio del café había logrado fortalecer la economía colombiana, que ahora, capitalizada, buscaba diversificar su aparato productivo (Palacios, 1979). Esta situación fue impulsada principalmente por la pérdida de los mercados externos que generaron la Gran Depresión (1929) y la Segunda Guerra Mundial (1939-1945) (Cárdenas, Ocampo, \& Thorp, 2003).

Estas circunstancias marcaron los inicios de uno de los procesos de industrialización más constantes que vivió el país, en simultáneo con otras naciones latinoamericanas que se embarcaban en proyectos similares, a los que se denominó ISI (Industria para la Sustitución de Importaciones) (BulmerThomas, 1997). Estos cambios fueron de la mano con procesos de

\footnotetext{
*Artículo tipo 3: de revisión según categoría de Colciencias.

** Historiador de la Universidad del Valle, miembro del grupo de investigación Nación Cultura Memoria y del grupo seminario de Historia Cultural del Departamento de Humanidades de la Universidad Icesi. Coordinador del tomo de historia política, del libro de Historia de Cali siglo XX. Actualmente es joven investigador de Colciencias con el proyecto "Transformaciones en la vida pública de Cali con la irrupción del gaitanismo", del cual se deriva el presente artículo. El autor agradece a sus colegas del Seminario por sus valiosos comentarios durante la etapa final del presente texto. Email: estebanmorera@gmail.com.
} 
urbanización acelerados, que estaban reconfigurando las diferentes ciudades del país y el continente (Zambrano \& Bernard, 1993).

Al tiempo que iban cambiando las condiciones económicas del país, la política iba tomando nuevas características debido a que sus actores se habían transformado sustancialmente. La irrupción de nuevos sujetos políticos iba propiciando la aparición de nuevas prácticas y movimientos ${ }^{1}$. La cultura política colombiana le estaba abriendo paso a una política de masas, donde los medios de comunicación (radio y prensa) ${ }^{2}$ se convirtieron en los principales canales de difusión de las ideas políticas. Este sería el escenario propicio para el surgimiento de fenómenos populistas, y en el caso colombiano, para el surgimiento del gaitanismo, un fenómeno de masas que reconfiguró el campo social e impuso una pausa a la cultura política tradicional, al bipartidismo decimonónico.

En la medida que el debate se iba ampliando, el discurso político iba tomando características más radicales y sectarias ${ }^{3}$. El debate político encontró en la descalificación del oponente la forma más usual de desarrollar los discursos, y la violencia se fue convirtiendo en el único medio de dirimir las diferencias. El asesinato de Gaitán fue una de las más significativas expresiones de esta dinámica. Si bien es cierto, la muerte del líder político no es la génesis del fenómeno de La Violencia, su asesinato representó para la vida política del país un punto de agudización de la más devastadora barbarie.

\section{La Violencia como campo de estudio}

La Violencia como fenómeno político no se puede definir como un simple enfrentamiento entre liberales y conservadores, o como una expresión diáfana de la lucha de clases. Dentro de este fenómeno subyacen pugnas de diversa naturaleza. Si bien es cierto, la Violencia se expresa como una degradación de la cultura política bipartidista, vemos en ésta enfrentamientos entre clases dominantes, entre clases dominantes y el movimiento popular, y expresiones del vandalismo y bandidismo (Sanchéz Gómez, 2008), características que variaban de región en región.

El balance de los estudios que se ocupan del fenómeno de la violencia política que se desarrolló entre los años cuarenta y cincuenta, muestra como estos encontraron un punto de partida en la obra clásica de Germán Guzmán, Orlando Fals Borda y Eduardo Umaña, La violencia en Colombia (1962). Las

\footnotetext{
${ }^{1}$ Uno de los trabajos más recientes que se realizan sobre este periodo y que de manera muy prolija indaga sobre las sociabilidades obreras en la ciudad de Cali, a través de los congresos obreros que se realizan en la ciudad, es la tesis de pregrado del historiador Camilo Serrano (Serrano Corredor, 2010).

${ }^{2} \mathrm{La}$ aparición de la radio representó un proceso de transformación cultural muy interesante, que necesariamente va impactar en sectores como el político de manera significativa. Por eso es un tema que merece un amplio desarrollo, a continuación alguna bibliografía que se pude revisar al respecto: Castrillón Gallego (2009), Castellanos (2001), Silva (2005), Téllez Benítez (1974). El autor agradece a Catalina Castrillón por su colaboración en la introducción a este tema.

${ }^{3}$ El estudio de estos aspectos es más visible en las elecciones de 1946. Existe una bibliografía disponible que hace amplia referencia a este proceso, uno de los más estudiados en la historia política del país: Fluharty (1957), Green (1996), Hartlyn (1993), Henderson (2006), Oquist (1980), Payne (1968), Schoultz (1972), Weiss (1968).
} 
tres visiones condensadas en este libro, se constituyeron en la primera conceptualización de La Violencia como un fenómeno nacional, abriendo un campo de estudio que se convirtió en el más profundizado en las ciencias sociales en nuestro país.

Otro de los avances significativos fue el trabajo de Paul Oquist (1980), que intentó dar una explicación al fenómeno desde el Estado y su "derrumbamiento parcial". Para Oquist en la medida que el estado colombiano fue creciendo, la pugna entre líderes liberales y conservadores por monopolizar sus dineros e influencia lo condujo a un colapso.

En este campo investigativo no hay un trabajo más completo e indispensable que el de Daniel Pécaut (2001), Orden y violencia (publicado por primera vez en 1987). En este texto el autor realiza un riguroso seguimiento a los orígenes de los diferentes fenómenos que coexistieron en La Violencia, entendida no como un hecho social, sino como uno de los momentos históricos en el cual confluyen los más diversos procesos de transformación social. Enunciándolo así, Pécaut realiza un exhaustivo análisis de cada uno de estos procesos, elaborando un cuadro teórico y metodológico.

Orden y Violencia se enfoca en la relación entre el Estado y la Sociedad Civil, mostrando cómo los procesos sociales y económicos de un país transforman el destino y las decisiones del sector político. En ese sentido, el autor explica La Violencia no como un hecho aislado de un movimiento popular, sino como un relevante acontecimiento social y político. Pécaut define al Estado como un Monopolio que enfrenta situaciones de violencia dentro y fuera de sus grupos sociales, donde el poder también se obtiene a través de la amenaza física y los delitos "legales".

Haciendo un retrato de la sociedad de la época, Pécaut muestra la conformación de las burguesías gracias al comercio del Café, hecho que propicia la entrada de la nación al mercado internacional. Así mismo, señala la estructuración de las clases urbanas, el protagonismo popular en los hechos políticos, la aparición del conflicto agrario, la figura de la Iglesia y la intervención del Estado.

Un detallado análisis de la función del Estado, es lo que le sirve a Pécaut para enmarcar la intervención del pueblo en el contexto político. La primera parte concluye con la exploración sobre las causas del detrimento del entorno social y político, paralelo a un conflicto de la república liberal durante la segunda presidencia del liberal López Pumarejo. La segunda, trata del auge del populismo, de las estrategias gaitanistas y del papel que desempeña el movimiento sindical. Tras referirse a los sucesos del 9 de abril de 1948, la obra culmina en un capítulo específico sobre la Violencia (1948-1953). Orden y violencia, al igual que Violencia, conflicto y política en Colombia de Paul Oquist, construyen fuertes entramados teóricos sobre la violencia, que le dan paso a la más amplia diversidad de estudios sobre el tema ${ }^{4}$.

\footnotetext{
${ }^{4}$ Otro amplio balance sobre la historia colombiana, que incluye el tema de la Violencia dentro de un análisis en un arco temporal mucho más amplio es el que realiza Marco Palacios en Entre la legitimidad y la violencia. En este libro el autor muestra los cambios económicos del país a partir de las imágenes
} 


\section{La Violencia y los estudios regionales}

La Violencia es sin lugar a dudas, uno de los temas más abordados en la historiografía nacional, y dependiendo de la naturaleza de cada investigación, se cuenta con una gran variedad de posibilidades bibliográficas. Para el caso de los estudios regionales, estos comienzan a contar con una gran relevancia con la aparición en escena del trabajo de Gonzalo Sánchez.

En Los días de la revolución (Sánchez Gómez, 1983) realizó un trabajo de observación general, sobre los diferentes hechos que sucedieron en las diferentes regiones del país. Aunque en este texto no encontramos mayores detalles ni desarrollos complejos sobre lo sucedido en las regiones, el aporte del profesor Gonzalo Sánchez muestra un camino de posibilidades de investigación en la provincia, al mostrar cómo la naturaleza de la violencia variaba de región en región. Si bien es cierto que previamente ya se habían realizado estudios regionales sobre la materia, el merito de Sánchez es que logra ver La Violencia como un todo en el que convergían fenómenos de diversa naturaleza.

Los avances de los estudios regionales en este campo, han contado con algunas experiencias notables, como son los casos de los trabajos de Jaime Arocha (1980) y Carlos Ortiz (1985) para el Quindío, Darío Fajardo (1977) y James Henderson (1985) para el Tolima, John Green (1994) para la Costa Atlántica y la región del río Magdalena, y uno de los más recientes (en ser publicado, ya que se deriva de su tesis doctoral [Roldán, 1992]) fue el estudio de Mary Roldán (2003) sobre la experiencia en Antioquia.

Otra de las obras que vale la pena resaltar, es quizás el más importante ejercicio de microhistoria con el que cuenta la historiografía nacional, Mataron a Gaitán de Herbert Braun (2008). Este texto invita además a la reflexión disciplinaria respecto al papel que debe jugar el relato dentro del trabajo historiográfico. Uno de los principales logros de Braun es ocultar las complejidades estructurales y teóricas, en una fluida narración que detalla la vida pública de Bogotá en la década de los cuarenta.

En Mataron a Gaitán, podemos observar el ascenso del pueblo al escenario político, a partir del Gaitanismo, narrando los hechos históricos que agudizan el fenómeno de la Violencia, comenzando el 9 de abril con el Bogotazo, el surgimiento del movimiento sindical hasta 1953. Con un estilo novedoso, el autor utiliza una amplia gama de fuentes documentales con: entrevistas, discursos políticos, prensa, documentos judiciales, literatura de novelas e informes oficiales. El autor caracteriza, describe con magnificencia, la vida pública bogotana de la década de 1940. Según las memorias, dichos sucesos

Coloniales rurales que sugerían marcadas diferencias entre ricos y pobres en un individualismo agrario y poder sobre la servidumbre. Se extiende en el relato hasta mediados del siglo XX, para describir a un país pobre que cuya configuración se deriva hacia una urbanidad de casas de invasión y subempleo y luego le da la bienvenida a la cultura popular, con música y televisión. El libro concluye con la etapa del derrumbe, como denomina su autor, a la corrupción de las elites, la ausencia de ley de los pobres, el desencanto político en relación las clases medias, el desarrollo acelerado del crimen en la calle y la reacción del gobierno a través de lo que llaman seguridad nacional (Palacios, 1995). 
son fundamentales para comprender la situación anterior y actual del país en relación con la política y con los movimientos populares.

En esta narración además se describe la carrera política de Jorge Eliécer Gaitán y su representación como eslabón que logra unir al pueblo con el estado, al ser admirado por las masas por ser de extracción humilde. Así mismo, Braun critica al régimen político bipartidista liberal-conservador (al que denomina convivialista), y muestra cómo este atraviesa una fuerte crisis a partir de la muerte del caudillo.

Desde este momento quedaba clara la importancia de que el estudio del fenómeno tuviese un desarrollo mucho más regional, ya que la naturaleza de la violencia contaba con características muy diferentes en cada una de las regiones del país. El trabajo de Braun, a pesar de haberle dado precisión empírica a trabajos como los de Oquist y Pécaut, limitó su mirada sobre los hechos acaecidos en la capital de la república.

\section{El gaitanismo en la ciudad de Cali}

En la primera mitad del siglo XX, Cali fue una de las ciudades que se vio impactada con intensidad por las transformaciones que experimentaba la sociedad colombiana, y fue uno de los lugares que vivió más de cerca el fenómeno gaitanista, a la luz de los resultados electorales. Cali, para la fecha, era un escenario que experimentaba diversos procesos de transformación, sintió de manera vigorosa la irrupción del gaitanismo y padeció las consecuencias de los hechos desencadenados el 9 de abril $^{5}$.

Desde la década de 1920 la ciudad experimentaba un ambiente de agitación política y de fortaleza de los sectores obreros (Archila Neira, 1991, págs. 435-446). Uno de los principales actores de las protestas obreras en esta ciudad, Ignacio Torres Giraldo (2004, pág. 188), quien comenta que Cali era "base de primera en todo movimiento de masas en el país, pueblo libérrimo con dirigentes que surgen de su propia entraña y por consiguiente plaza de las izquierdas colombianas". Las razones son múltiples: la diversidad poblacional, el fuerte crecimiento urbano, la posibilidad de acceso a los medios masivos de comunicación y la posición estratégica que había adquirido la ciudad (entre otras), habían cambiado tanto su morfología como la de los habitantes que frecuentaban sus calles, creando nuevos sujetos políticos, con nuevas expresiones. La calle como espacio de manifestación de las ideas, espacio de difusión y de exhibicionismo político, alcanzó su cénit con el gaitanismo cohesionando gran parte de estas luchas (Williford, 2010).

\footnotetext{
${ }^{5}$ Estas transformaciones se pueden observar en diversos trabajos que han estudiado la sociedad caleña en su conjunto, como el trabajo de el profesor Edgar Vásquez Benítez (2001); desde la formación industrial de la ciudad y el nacimiento de una élite económica, que al mismo tiempo ejerció como élite política en el trabajo del profesor Jairo Henry Arroyo (2006), al igual que la conformación de la ciudad-región bajo un proyecto de desarrollo con características modernizantes, en el muy valioso y poco explorado trabajo del profesor Oscar Almario García (1994); o desde la trasnformación urbana que estudia el profesor Jacques Aprile-Gniset (1992).
} 
Para el caso del Valle del Cauca, el trabajo del profesor Sánchez (1983) podría calificarse de escaso, y más aún para lo sucedido en Cali. Este libro además de contar con una corta extensión, se dedica a hacer un reducido inventario de lo acontecido en la ciudad, horas posteriores al asesinato de Gaitán. Durante mucho tiempo esta fue la única voz al respecto. Los estudios sobre el gaitanismo y el 9 de abril en la ciudad, se limitaban a las tres o cuatro páginas que le había ofrecido el profesor Sánchez y un artículo, también muy corto, que realizó Darío Betancourt (1987).

Algunos avances muy valiosos se realizaron en libros que abordaban problemas de investigación diversos y que le daban relevancia al desarrollo de los diferentes procesos en la ciudad. Hay que tener en cuenta que la ciudad de Cali y el departamento del Valle del Cauca, contaban con características muy singulares frente a las otras regiones del país, y por esta razón fue tenida en cuenta en numerosos estudios. Como son los casos de el ya mencionado Orden y violencia, La ofensiva empresarial ${ }^{6}$ (Saenz Rovner, 1992), Historia del partido comunista (Medina, 1980), El porvenir del pasado: Gilberto Alzate Avendaño, sensibilidad leoparda y democracia (Ayala Diago, 2007), entre otros.

Dentro de las diferentes monografías de grado, existen ciertos avances que por no haber sido publicados pueden pasar inadvertidos. Hasta el momento, entre los estudios sobre este periodo en la ciudad cabe mencionar la tesis de Ángela María Hoyos Mazuera (1984) Gaitanismo y la prensa en Cali (19461948), donde se realiza un análisis de las posturas de los principales rotativos de prensa local escrita. En este trabajo, la autora realiza un estudio de los editoriales y las notas periodísticas, para las elecciones de 1946 y los dos primeros años del gobierno de Mariano Ospina Pérez. Por otro lado, la tesis de María Eugenia Díaz y Graciela Lozada (1994) Partidos y Gobierno en el Valle del Cauca 1945-1950, muestra la conformación de los grupos políticos más dominantes en la ciudad, así como algunos acercamientos a lo que fue el gaitanismo en Cali. Finalmente, dentro de las tesis de pregrado más cercanas a nuestro objeto de estudio, encontramos Antecedentes y consecuencias del 9 de abril en Cali y otras regiones del Valle del Cauca (Cortés Arango \& Romero Girón, 1990), en la cual se describen los diferentes acontecimientos que se presentaron el día de la muerte de Gaitán, así como los que se presentaron en los días posteriores.

En este tipo de trabajos (tesis de pregrado), encontramos principalmente estudios puntuales sobre diferentes elementos de la sociedad, pero que se presentan relativamente aislados, situación que atenta contra la clara observación del objeto de investigación.

\footnotetext{
${ }^{6}$ Me parece muy importante hacer mención de este libro, el cual es uno de los más novedosos estudios sobre la Violencia en Colombia, una de las más innovadoras miradas de este fenómeno. La ofensiva empresarial explicita la consciencia adquirida por el gremio empresarial Antioqueño, con mayor peso en el área de textiles, de que se hacía imperioso defender y desarrollar los intereses creados a propósito de sus prolíficas actividades económicas que con el tiempo fueron diversificándose. Así las cosas, el texto expone con claridad las estrategias de las que supieron valerse los mencionados capitalistas construyendo discursos que versaban sobre la importancia del fortalecimiento de la industria nacional. El capital, entre otras cosas, sirvió para desarrollar un músculo discursivo impulsado por medios de comunicación e incluso intelectuales, que logró abrirse paso entre la opinión pública (Saenz Rovner, 1992).
} 
En cuanto a las tesis de maestría, encontramos el trabajo de Carlos Andrés Charry (2004), investigación de la que ha derivado diversos artículos de revistas (Charry Joya, 2006) (2009) y la publicación de un libro en el año 2010 (Charry Joya, 2010).

Este trabajo es el último y más significativo avance de los estudios del gaitanismo en la ciudad, además de ser el primero en afrontar el fenómeno de 9 de abril reuniendo diferentes elementos de la sociedad para explicar lo que él denomina "un proceso social". Cuenta con un extenso recorrido de acontecimientos, que parten desde la república liberal, y que el autor utiliza para elaborar la construcción de este "proceso social", y de sustento para su intento de aplicar la teoría de Norbert Elias, sobre los grupos "establecidos y marginados".

Sin embargo, encuentro en este trabajo dificultades que me parece importante resaltar. Si bien es cierto que el propio autor reconoce que su trabajo cuenta con limitaciones debido a la metodología que aplicó para el abordaje de las fuentes, planteando una inevitable "servidumbre de las fuentes" (acuñando la frase de Renán Silva en $A$ la sombra del Clío) a la cual estamos sometidos los historiadores, creo que esto no puede ser un escudo para suprimir los avances de la historia crítica. Más allá de que gran parte de nuestras investigaciones están determinadas por las fuentes, esto no se puede convertir en una situación inmutable a la cual el investigador se tenga que resignar, menos aún en la historia del siglo $\mathrm{XX}$ donde existen diversas posibilidades documentales.

Considero que es un gran aporte haber explorado los archivos de la gobernación, sin embargo veo que esto lo ha llevado cometer inexactitudes, que pueden ser fruto de la reproducción exclusiva de una fuente oficial. Un síntoma de estas imprecisiones es el mapa político que construye, donde figuras relevantes de la política local como Alfonso Barberena se expone como si hubiese sido comunista ${ }^{7}$, siendo este uno de los más reconocidos políticos liberales de la ciudad, un hecho que evidencia el desconocimiento que tiene este trabajo por movimientos como la lucha por la vivienda popular y la lucha por la municipalización de las empresas de servicios públicos que libró Barberena junto al comunista Julio Rincón.

Reconociendo la formación del autor, y que el trabajo que este realiza es basado en una tesis para su maestría en sociología, creo sin embargo que sus dificultades no solo son del orden de la documentación utilizada. Pienso también que hay que hacer un análisis valorativo sobre la relevancia que puede tener aplicar una teoría como la de Norbert Elías a un proceso como el que vivió el gaitanismo en la ciudad y el departamento. Charry plantea que es posible hacer una lectura de los sucesos desde la perspectiva dada por la

\footnotetext{
${ }^{7}$ Este error es recurrente en toda su obra, situación que llama la atención, ya que en su primer publicación en el 2006 aparece esta falla, y esta se reproduce hasta su última en el 2010 (p. 186). Esto es sin lugar a dudas un síntoma del limitado diálogo académico que hay sobre el tema, dificultad adicional que ha tenido el profesor Charry para el desarrollo de toda esta obra, que a pesar de ya contar con cuatro años no evidencia ningún avance significativo frente a su concepción original.
} 
teoría de los procesos sociales y puntualmente desde lo referido a las relaciones entre establecidos y marginados, que desarrolla Elías.

Resulta difícil encontrar el progreso analítico en este tema tras la aplicación de una teoría como la de Elías, ya que si bien es cierto, la sociedad colombiana era para ese entonces una sociedad excluyente, entender este proceso como tal no le entrega ninguna particularidad adicional a lo que se encontraba previamente en el campo investigativo. Ahora bien, una vez Charry comienza a hacer un desarrollo del proceso entendiéndolo bajo esta lógica, no hay en el texto una definición clara de cómo se conforman estos dos grupos sociales (establecidos y marginados), ya que si se mira solo bajo la óptica de quienes detentan el poder con respecto de quienes están excluidos de él, necesitaríamos entender cuáles elementos de la sociedad están librando esta pugna.

Pienso, sin embargo, que las dificultades que presenta el trabajo del profesor Carlos Andrés Charry, son el síntoma del limitado diálogo académico que existe sobre el tema en la ciudad. Más allá de que el fenómeno de la violencia es quizás uno de los temas más tratados en la historiografía nacional, la necesidad de hacer desarrollos más regionales sobre este problema (que se evidenció en la década de 1980), no abrió un amplio debate en nuestra región, situación que da mayor importancia al trabajo desarrollado por Charry, que a pesar de las dificultades, ha logrado impulsar una serie de nuevas investigaciones, que se esfuerzan por cubrir vacíos específicos del campo investigativo como lo son los trabajos de Ana Lorena Yara (2011), que trata de los acontecimientos acaecidos en las poblaciones de Cali y Puerto Tejada, y el trabajo de Diana Gómez Charry (2011) que hace un análisis sobre la prensa caleña durante el gobierno conservador de Ospina Pérez y el primer año de Laureano Gómez.

El cometido, por tanto, del presente artículo, es retomar el debate sobre este importante tema en la ciudad, incluyendo estos nuevos trabajos, en los cuales se encuentra el que realizó el autor del presente artículo como tesis de pregrado (Morera Aparicio, 2011), sobre el gaitanismo como un fenómeno urbano y populista, en el cual se intenta hacer un mapa de la vida pública caleña, para entender qué transformaciones sufrió la ciudad con la irrupción del gaitanismo en la vida política nacional y la posterior desaparición del caudillo. En este trabajo se dedica a mostrar cómo se constituía la vida pública de la ciudad, cómo se organizaban los diferentes grupos y cuál era su relación con el espacio público. Teniendo en cuenta que para este momento la calle era el principal centro de actividad política, la irrupción de la violencia, así como la llegada de la radio, van a ir transformando la forma de desarrollar la política en la ciudad.

Una de las ventajas de los estudios sobre el siglo XX es la gran variedad de fuentes documentales con las que se puede contar. Para la década de 1940 en la ciudad de Cali, eran tres los principales diarios: el periódico conservador Diario del Pacífico, liderado por la familia Borrero Olano; Relator, diario liberal santista de la familia Zawadsky; y El Crisol, un periódico liberal, lopista, de propiedad del líder político Rafael Isidro Rodríguez, quien se conoció bajó el 
pseudónimo de Placido Soler. Adicionalmente es importante tener en cuenta el semanario El Gato, que puede ser una importante fuente de información al momento de estudiar la actividad política de la región. El Gato también contaba con una importante producción de caricaturas, que era una de las principales cualidades por las que era reconocido ${ }^{8}$. Otro diario que puede dar un aporte significativo es el bogotano El Tiempo, ya que contó con un corresponsal fijo durante este periodo, y en ocasiones, por no obedecer a las dinámicas de la política local, presentaba noticias que no eran publicadas en los diarios locales. Este periódico bogotano se puede encontrar completo en línea, por su parte, la prensa local se puede consultar con facilidad en la hemeroteca de la Biblioteca Departamental "Jorge Garcés Borrero" y en el área cultural del Banco de la República de Cali, donde se encuentran muchos ejemplares microfilmados.

De fuentes oficiales también hay un importante acerbo documental, ubicado principalmente en el Archivo de la Gobernación del Valle del Cauca y en el Archivo Histórico de Cali. De la misma manera existen archivos fotográficos, estos son el Archivo fílmico y fotográfico del Valle del Cauca y una nueva colección de fotografías que adquirió el Banco de la república del fotógrafo Alberto Lenis Burckhardt.

Como se puede observar, las condiciones están dadas para que este campo de estudio amplié su discusión, en los últimos años se ha dado un primer paso con la incursión de nuevos trabajos, que son la muestra de que el estudio del gaitanismo en la ciudad ha comenzado una nueva etapa, sin embargo, es fundamental que estos no se desarrollen como universos individuales con una retroalimentación limitada, que impida que cada esfuerzo investigativo realice un aporte óptimo a la comprensión del fenómeno en la ciudad.

\section{REFERENCIAS BIBLIOGRÁFICAS}

Almario García, O. (1994). La configuración moderna del Valle del Cauca, Colombia, 1850-1940. Cali: Cecan.

Aprile-Gniset, J. (1992). La Ciudad Colombiana: Siglo XIX y Siglo XX. Bogotá: Banco Popular.

Archila Neira, M. (1991). Cultura e identidad obrera, Colombia 1910-1945. Bogotá: Cinep.

Arroyo Reina, J. H. (2006). Historia de las practicas empresariales en el Valle del Cauca: Cali 1900 - 1940 . Cali: Universidad del Valle.

Arocha, J. (1980). La Violencia en el Quindío. Bogotá: Tercer Mundo.

\footnotetext{
${ }^{8}$ Actualmente el historiador Rubén Armando Hurtado se encuentra desarrollando una interesante investigación sobre la caricatura de este semanario. Probablemente al momento de la publicación de este artículo ya se puede contar con ese trabajo.
} 
Ayala Diago, C. A. (2007). El Porvenir del Pasado: Gilberto Alzate Avendaño, Sensibilidad leoparda y democracia. La derecha colombiana de los años treinta. Bogotá: Fundación Gilberto Alzate Avendaño.

Betancourt, D. (1987). "El 9 de abril en Cali y en el Valle". Anuario colombiano de Historia Social y de la Cultura , 15, 273-278.

Braun, H. (2008). Mataron a Gaitán. Vida pública y violencia urbana en Colombia. Bogotá: Aguilar.

Bulmer-Thomas, V. (1997). "Las economías latinoamericanas, 1929-1939". En L. Bethell, Historia de América Latina (pp. 3-82). Barcelona: Cambridge University Press, Crítica.

Cárdenas, E., Ocampo, J. A., \& Thorp, R. (2003). La era de las exportaciones latinoamericanas. México : Fondo de Cultura Económica.

Castellanos, N. (2001). "La civilización del iletrado. El proyecto ilustrado de la radiodifusión en Colombia 1929-1940". En J. I. Bonilla, \& G. Patiño, Comunicación y política. Viejos conflictos, nuevos desafíos. Bogotá: CEJA.

Castrillón Gallego, C. (2009). "La radio educadora: solución para una patria inculta. La actividad radial en Colombia, 1930-1940". En D. L. Ceballos Gómez, Prácticas, territorios y representaciones en Colombia, 1849-1960 (págs. 129-145). Medellín: Universidad Nacional.

Charry Joya, C. A. (2004). Los sucesos del 9 de abril en Cali: liminalidad y transformaciones del poder social en el Valle del Cauca (1928-1949). (Tesis de maestría en Sociología). Cali: Universidad del Valle.

Charry Joya, C. A. (2006). "El 9 de abril en Cali: cambio social, poder y liminalidad en el Valle del Cauca". Anuario colombiano de Historia Social y de la cultura (33), 143-182.

Charry Joya, C. A. (2009). "El impacto de 9 de abril en Cali y el Valle del Cauca". Revista CS , 55-89.

Charry Joya, C. A. (2010). Los sucesos del 9 de abril en Cali y el Valle del Cauca, historia de un proceso social. Cali: Universidad Libre.

Cortés Arango, F., \& Romero Girón, G. (1990). Antecedentes y consecuencias del 9 de abril en Cali y otras regiones del Valle del Cauca. (Tesis pregrado en Historia). Cali: Universidad del Valle.

Díaz, M. E., \& Lozada, G. (1994). Partidos y gobierno en el Valle del Cauca 1945-1950. (Tesis para licenciatura en ciencias sociales). Cali: Universidad del Valle.

Fajardo, D. (1977). "La violencia y las estructuras agrarias en tres municipios cafeteros del Tolima: 1936-1970". En El agro en el desarrollo histórico colombiano. Bogotá: Punta de Lanza.

Fluharty, V. (1957). Dance of the millons: military rulesahd the social revolution en Colombia, 193-1956. Pittsburgh: University of Pittsburgh. 
Gómez Charry, D. (2011). La prensa en Cali, tras el asesinato de Jorge Eliécer Gaitán y el advenimiento de la violencia bajo el gobierno conservador (1946-1951). (Tesis de Licenciatura en Historia). Cali: Universidad del Valle.

Green, J. (1994). Popular mobilization in Colombia : the social composition, ideology, and political practice of Gaitanismo on the Atlantic Coast and Magdalena river, 1928-1948. (Tesis Doctor of Philosophy). Austin: The University of Texas at Austin.

Green, J. (1996). "Mujeres radicales, el voto y la participación femenina en la política gaitanista". Anuario colombiano de Historia Social y de la Cultura , 23, $159-170$.

Hartlyn, J. (1993). La política del régimen de coalición: la experiencia del Frete Nacional en Colombia. Bogotá: Tercer Mundo.

Henderson, J. D. (2006). La modernización en Colombia: los años de Laureano Gómez, 1889-1965. Medellín: Universidad de Antioquia.

Henderson, J. D. (1985). When Colombia bled: a history of the 'Violencia' in Tolima. University: University of Alabama Press.

Hoyos Mazuera, Á. M. (1984). Gaitanismo y la prensa escrita en Cali (19461948). (Tesis de pregrado en Historia). Cali: Universidad del Valle.

Medina, M. (1980). Historia del Partido Comunista. Bogotá: CEIS.

Morera Aparicio, E. (2011). Vida pública en Cali y el impacto del gaitanismo en la ciudad (1945-1950). (Tesis de pregrado en Historia). Cali: Universidad del Valle.

Oquist, P. (1980). Violence, conflict \& politics in Colombia, on the Violencia of the 1950s. New York: Academic press.

Ortiz Sarmiento, C. M. (1985). Estado y subversión en Colombia. La Violencia en el Quidío años 50. Bogotá: Cider-Cerec.

Palacios, M. (1979). El café en Colombia (1850-1970). Bogotá: Presencia.

Palacios, M. (1995). Entre la legitimidad y la violencia, Colombia 1875-1994. Bogotá: Norma.

Payne, J. (1968). Patterns of conflict in Colombia. New Haven: Yale University Press.

Pecaut, D. (2001). Orden y Violencia: Evolución Socio-Política de Colombia Entre 1930 y 1953. Bogotá: Norma.

Roldán, M. (2003). A sangre y fuego: la violencia en Antioquia, Colombia, 19461953. Bogotá: ICAH.

Roldán, M. (1992). Genesis and evolution of La Violencia in Antioquia. Colombia (1900-1953). (Tesis doctoral). Harvard University.

Saenz Rovner, E. (1992). La ofensiva empresarial. Bogotá : Tercer Mundo. 
Sanchéz Gómez, G. (2008). Guerra y política en la sociedad colombiana. Bogotá: Aguilar.

Sánchez Gómez, G. (1983). Los días de la Revolución: gaitanismo y el 9 de abril en Provoncia. Bogotá: Centro cultural Jorge Eliecer Gaitán.

Schoultz, L. (1972). Urbanization and changing voting patterns. Politicalscience quarterly (87), 22-45.

Serrano Corredor, C. E. (2010). Il Congreso de la Confederación de Trabajadores de América Latina, Cali, 1944: ¿Institucionalización, inclusión o cooptación del Movimiento Obrero?. (Tesis de licenciatura en Historia). Cali: Universidad del Valle.

Silva, R. (2005). República liberal, intelectuales y cultura popular. Medellín: La Carreta.

Téllez Benítez, H. (1974). Cincuenta años de radiodifusión en Colombia. Medellín: Bedout.

Torres Giraldo, I. (2004). Anecdotario. Cali: Universidad del Valle.

Vásquez Benítez, E. (2001). Historia de Cali Siglo 20: Sociedad, economía, cultura y espacio. Cali: Artes Graficas del Valle.

Weiss, A. (1968). Tendencias de la participación electoral en Colombia, 19351966. Bogotá: Universidad Nacional de Colombia.

Williford, T. (2010). "Desfiles y Antorchas: Las manifestaciones físicas de la política en Colombia en los años 30 y 40". Memorias XV Congreso Colombiano de Historia. Bogotá.

Yará, A. L. (2011). 9 de abril de 1948 en Pueto Tejada y Cali como un acontecimiento histórico. (Tesis de pregrado en Historia). Cali: Universidad del Valle.

Zambrano, F., \& Bernard, O. (1993). Ciudad y territorio: Procesos de poblamiento en Colombia. Bogotá: Tercer Mundo.

Recibido: Octubre 25 de 2011

Aprobado: Mayo 7 de 2012 